\title{
RGB Image Reconstruction Using Two-Separated Band Reject Filters
}

\author{
Muthana H. Hamd \\ Computer/ Faculty of Engineering, Al Mustansirya University \\ Baghdad, Iraq
}

\begin{abstract}
Noises like impulse or Gaussian noise could be easily removed and recovered in the spatial domain by applying mean or median convolution. Structural noise, like periodic has a global degradation effect. This degradation is looking like bars that cover the image. This paper developed a new approach that detect the degradation; recover it; and hence reconstruct the original color image. The two dimensions discrete Fourier Transform is applied to isolate, block, and replace some particular frequencies (degradation bands) using auto detection and recovery procedure, it applies two separated band reject filters to avoid blocking the true bands like what is resulted in Ring filter. It tries locating the periodic noise (two spikes) and the four degraded lines using a powerful searching algorithm in the frequency domain. Unlike, Ring and Notch filter, the Two-Circle method is smart enough to predict the size of the spike, so a suitable band rejected filter should be selected to replace only degraded bands with approximation values instead of zeroes. The Peak Signal to Noise Ratio (PSNR) vs. periodic frequency relation is applied to find the quality of measurements of the reconstructed and cleaned colored image. So, the quality of three methods is calculated and compared. All testing results show that the two separated band reject filters method satisfied maximum $\mathrm{dB}$ results and minimum frequency change to the steady state values than other two methods.
\end{abstract}

Keywords: Periodic Noise, BRF, Ring Filter, Notch Filter, DFT

\section{INTRODUCTION}

Noise can be defined as any degradation or error in the image pixel value, which is caused by an external disturbance. When this disturbance becomes periodically rather than random disturbance, a corruption in image signal is obtained as bars over the image. The electromagnetic field of electric motor is an example of repeating nature that causes degradation over transmitting image. The two dimension discrete Fourier transform (2D DFT) is applied to process and decrease the degradation effect using the new transformed image features. The relatively high change of image frequency in spatial or data domain would be transformed into two spikes in image frequency domain. The location of two spikes reflects the 
range of periodic noise frequency (bars) in spatial domain. Two approaches were used to remove or decrease this degradation, first one is the Band Reject Filter (BRF) or Ring filter and the second one is the Notch filter. This paper develops two auto detection and recovery procedures that acts as two separated BRFs with a combined Notch filter $[1,2,3]$.

They try removing and replacing only corrupted bands. The two spikes and their associated four lines are recovered by an approximation value other than zeroes. A comparison is made between these methods by finding the image qualification using Peak Signal to Noise Ratio (PSNR). This paper is organized as follows: Section 1 is an introduction, section 2, explains the main types of structured noise; section 3 illustrates the auto detection and recovery procedures that are used to detect and replace the unwanted bands with approximation values. The comparison between these methods is presented in section 4 . The last section presents the conclusion.

\section{STRUCTURED NOISE}

The structured noise is classified into stationary with a fixed amplitude, frequency, and phase or non-stationary, where the noise parameters are varying across the image. When this image signal is subjected to a periodic, rather than a random disturbance, image will be corrupted by periodic noise $[4,5]$. So periodic noise may occur in the image because of:

- Image equipment

- Network equipment

- External disturbance of repeating nature

The general trigonometric formula that causes bars over image is [5].

$$
\text { Noise }(\text { periodic })=\operatorname{sine}\left(\omega_{1} x+\omega_{2} y\right)+1
$$

Where:

$$
\begin{aligned}
& x, y \text { : are image plane pixel values } \\
& \omega_{1,2} \text { : Periodic noise frequencies }
\end{aligned}
$$

$\omega_{1}$ is responsible for horizontal shift of spikes along $x$-axis, and $\omega_{2}$ is responsible for vertical movement along $y$-axis. For $(256 \times 256)$ image, the movement scale is approximately from 0.1 which is closed to the image center (DC coefficients) towards outside at $\omega_{1,2} \approx 2.9$. If this degradation in equation 1 is injected in the RGB image planes, the process could be implemented for reach plane individually, by applying equation 2 .

$$
\mathrm{RGB}_{\text {noisy }}=\text { Periodic noise }+\mathrm{RGB}(\mathrm{r}, \mathrm{g}, \mathrm{b})
$$

\section{FREQUENCY DOMAIN NOISE REMOVAL}

Image transformation from spatial or data domain to the frequency domain could be implemented using the Two Dimension Discrete Fourier Transform (2D-DFT). Filtering or convolution in spatial domain is converted to the whole image and mask multiplication in 
frequency domain. Transforms to the new domain can obtain many new features like: conjugate symmetry, mirror, DC coefficients...etc $[6,7,8]$. The periodic noise frequency or bars would be represented as two shiny spikes in frequency domain. The two shiny spikes have maximum frequency complex value. If the spikes is detected and located, their associated two degraded lines will be detected and located also. If the frequency of periodic noise is low, the spikes would be closed to the DC coefficient (image center) and vice versa; they move out diagonally toward image plane edges when frequency of periodic noise is increased, see Fig. 1 and 2 . For a given noise frequency $\omega_{1}$ and $\omega_{2}$, the searching procedure is started looking for the two maximum values (spikes) diagonally, if founds a recovery procedures is beginning Ring and Notch filters usually replace the degraded bands with zeros, while auto detection and recovery procedure try approximating the degraded bands with a mean band value for spike's neighbour, as follows;

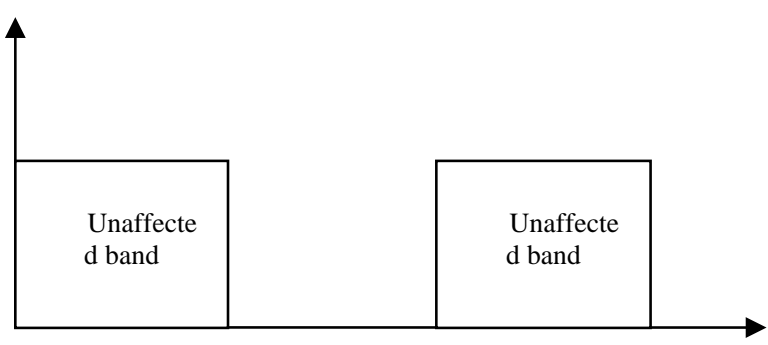

(a)

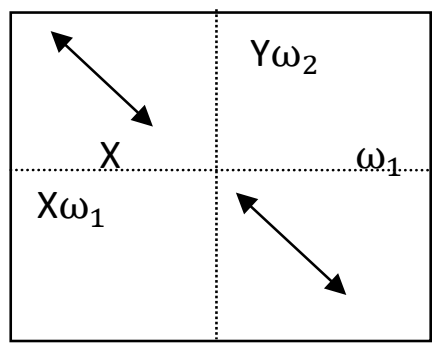

(b)

Figure 1: (a) spikes band reject filter, (b) periodic noise frequency movement

\section{AUTO DETECTION AND RECOVERY PROCEDURE}

The auto detection procedure starts finding the shiny spike which holds a maximum complex value in frequency band of quarter one. Once, it is located, the next step is to assign values instead of zeros. The new idea in this approach is the approximation value assignment. Instead of zeros substitution as in Ring and Notch filter, the Auto detection approach finds an approximation value as follows:

- $\quad \operatorname{app}_{\mathrm{s}}=\frac{\text { spike value }(\max )}{\sqrt{\text { spike value }}}$

- Associated spike lines

$$
\begin{aligned}
& a p p_{r w}=\frac{\sum_{i=1}^{M} R O W_{i}}{M} \\
& a p p_{c l}=\frac{\sum_{i=1}^{N} C O L_{i}}{N}
\end{aligned}
$$

Where,

$\operatorname{app}_{\mathrm{s}}$ : is the approximation value

$\operatorname{app}_{\mathrm{rw}}$ : is the approximation value of row vector

$\operatorname{app}_{\mathrm{cl}}$ : is the approximation value of column vector 


\section{QUALITY MEASURMENT AND COMPARISION}

Four $(256 \times 256)$ colored image are considered and tested by three periodic noise removal methods. First, images are injected with variant frequencies of periodic noise, ranging from $\omega_{1,2}=0.125$ to $\omega_{1,2}=2.9$. Then auto detection and recovery procedures results are tabulated in table 1, 2, and 3. These results belong to the Green.jpg image and it finds a relation between periodic noise frequency $\left(\omega_{1,2}\right)$ and image quality, (PSNR). The Two-Circle BRF results (PSNR vs. $\omega_{1,2}$ ) for all three tables are better and more stable than other two detection methods. That's because of smartness of the Two-Circle procedure where, it detects the size of spikes for each periodic noise value and a suitable band reject filter is chosen to block the degraded bands then change it with an approximation value as computed in equation 4. The PSNR qualifications for all three methods are represented in figure 3 . The Two-Circle BRF method satisfied rapid change and reached to the maximum $\mathrm{dB}$ and steady state value with $\omega_{1,2}=0.3$, i.e.; after 4 change of $\omega_{1,2}$. The Two-circle method is very suitable to remove small or large value of degradation, where, the area of the Two-circle BRFs is changed according the spike location. The closest to the DC coefficients, the smallest area of the Two-Circle filters, see Figure 2. Ring filter curve starts with $10.29 \mathrm{~dB}$ but it reaches to the maximum PSNR at $\omega_{1,2}=1.0$ as shown in figure 2 , it means that Ring method satisfy the steady state $(10.42 \mathrm{~dB})$ after 8 changes of $\omega_{1,2}$. Like Ring filter, Notch filter starts with initial value $(10.20 \mathrm{~dB})$ and it reaches to the steady state at $\omega_{1,2}=0.75$, i.e.; after 7 change of $\omega_{1,2}$. The four reconstructed and cleaned images are figured in Fig.4

Table 1. Ring Method

\begin{tabular}{|c|c|c|c|c|c|c|c|c|c|c|c|c|}
\hline & \multicolumn{10}{|c|}{ Ring Method, PSNR (dB) } \\
\hline$\omega_{1,2}$ & 0.125 & 0.15 & 0.175 & 0.2 & 0.3 & 0.4 & 0.5 & 0.75 & 1.0 & 1.75 & 2.5 & 2.9 \\
\hline Lenna & 7.00 & 7.01 & 7.02 & 7.02 & 7.03 & 7.03 & 7.03 & 7.04 & 7.04 & 7.04 & 7.04 & 7.04 \\
\hline Football & 10.98 & 10.98 & 10.99 & 10.99 & 10.99 & 10.99 & 10.99 & 11.00 & 11.00 & 11.00 & 11.00 & 11.00 \\
\hline Greens & 10.29 & 10.33 & 10.34 & 10.36 & 10.36 & 10.37 & 10.38 & 10.41 & 10.42 & 10.42 & 10.42 & 10.42 \\
\hline Peppers & 8.65 & 8.67 & 8.68 & 8.69 & 8.69 & 8.70 & 8.70 & 8.71 & 8.71 & 8.71 & 8.71 & 8.71 \\
\hline
\end{tabular}

Table 2. Notch Method

\begin{tabular}{|c|c|c|c|c|c|c|c|c|c|c|c|c|}
\hline & \multicolumn{10}{|c|}{ Notch Method, PSNR (dB) } \\
\hline$\omega_{1,2}$ & 0.125 & 0.15 & 0.175 & 0.2 & 0.3 & 0.4 & 0.5 & 0.75 & 1.0 & 1.75 & 2.5 & 2.9 \\
\hline Lenna & 6.98 & 6.99 & 7.00 & 7.01 & 7.03 & 7.03 & 7.04 & 7.04 & 7.04 & 7.04 & 7.04 & 7.04 \\
\hline Football & 10.95 & 10.97 & 10.97 & 10.98 & 10.99 & 11.00 & 11.00 & 11.00 & 11.00 & 11.00 & 11.00 & 11.00 \\
\hline Greens & 10.20 & 10.25 & 10.30 & 10.32 & 10.38 & 10.40 & 10.41 & 10.42 & 10.42 & 10.42 & 10.42 & 10.42 \\
\hline Peppers & 8.60 & 8.64 & 8.66 & 8.67 & 8.70 & 8.70 & 8.71 & 8.71 & 8.71 & 8.71 & 8.71 & 8.71 \\
\hline
\end{tabular}


Table 3. Two-Circle Method

\begin{tabular}{|c|c|c|c|c|c|c|c|c|c|c|c|c|}
\hline & \multicolumn{10}{|c|}{ Two Circle Method, PSNR (dB) } \\
\hline$\omega_{1,2}$ & 0.125 & 0.15 & 0.175 & 0.2 & 0.3 & 0.4 & 0.5 & 0.75 & 1.0 & 1.75 & 2.5 & 2.9 \\
\hline Lenna & 7.02 & 7.03 & 7.03 & 7.03 & 7.04 & 7.04 & 7.04 & 7.04 & 7.04 & 7.04 & 7.04 & 7.04 \\
\hline football & 10.98 & 10.99 & 10.99 & 11.00 & 11.00 & 11.00 & 11.00 & 11.00 & 11.00 & 11.00 & 11.00 & 11.00 \\
\hline Greens & 10.36 & 10.39 & 10.40 & 10.41 & 10.42 & 10.42 & 10.42 & 10.42 & 10.42 & 10.42 & 10.42 & 10.42 \\
\hline Peppers & 8.69 & 8.70 & 8.70 & 8.70 & 8.71 & 8.71 & 8.71 & 8.71 & 8.71 & 8.71 & 8.71 & 8.71 \\
\hline
\end{tabular}

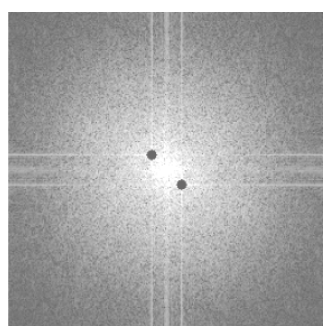

$\omega_{1,2}=0.15$

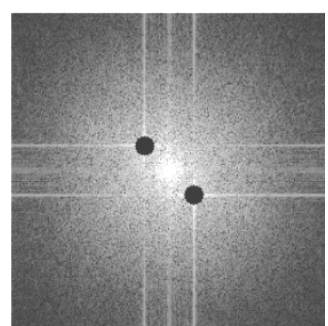

$\omega_{1,2}=0.2$

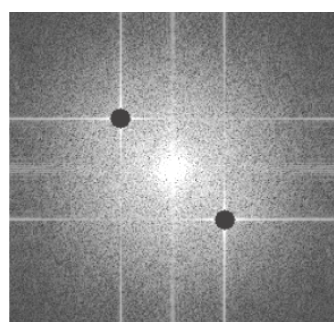

$\omega_{1,2}=1.75$

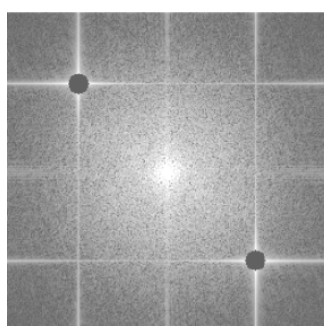

$\omega_{1,2}=2.5$

Figure 2. Auto detection and periodic noise removal procedure

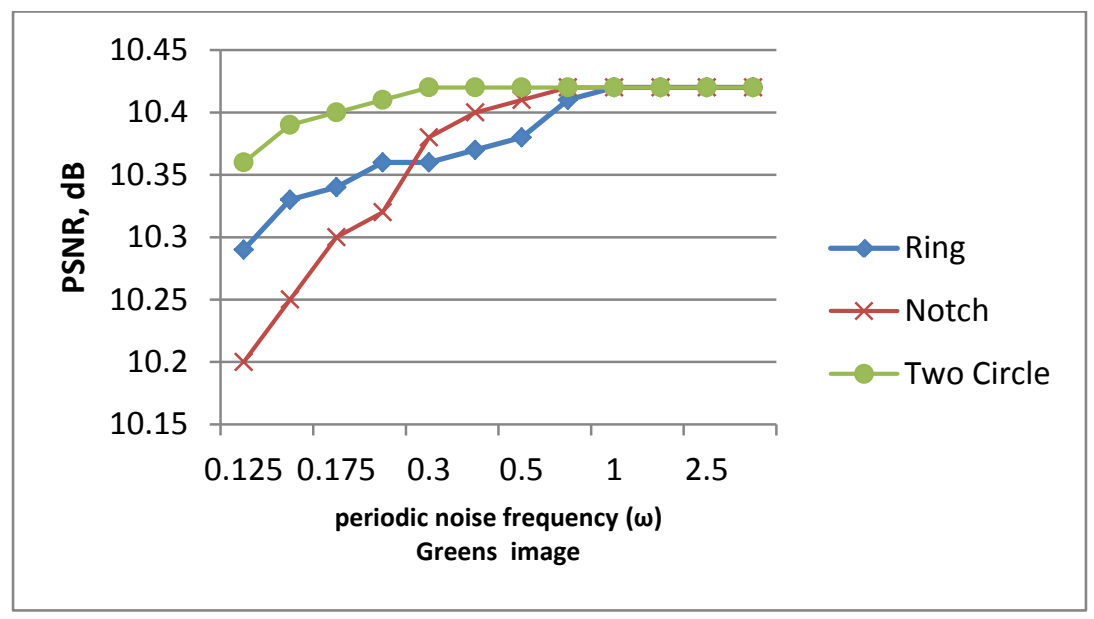

Figure 3. PSNR curves for three filter types 

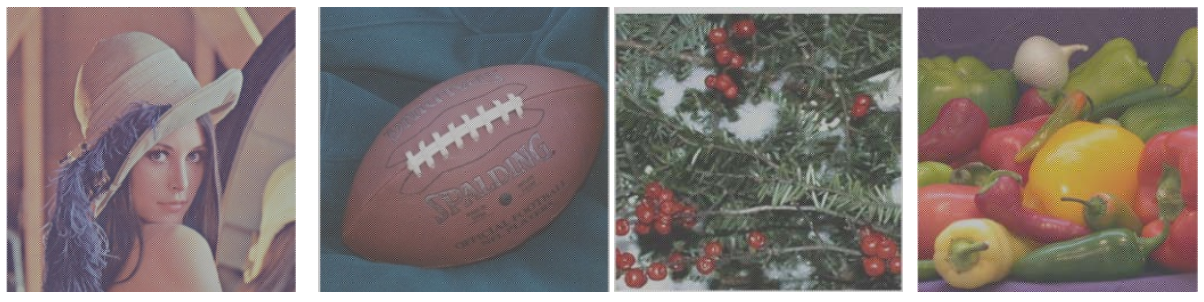

(a)
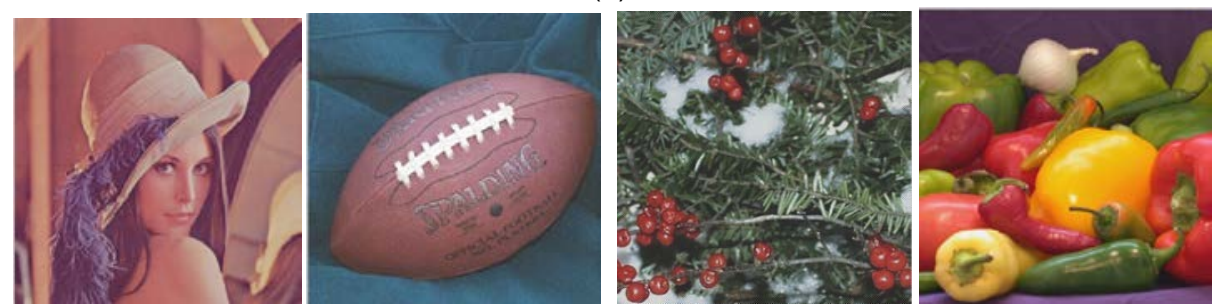

(b)

Figure 4. (a) noisy images, (b) reconstructed and cleaned images

\section{CONCLUSION}

Three auto detection and periodic noise removal methods have been developed and implemented to remove or decrease the degradations that could be resulted from an external disturbance like periodic noise. The two separated band reject filters are designed to block and replace the degradation frequency bands by approximation values after transforming noisy image into the 2D discrete Fourier transform. The two common methods: the Ring and Notch filters are modified to be auto detected and recovered procedure. All methods have been implemented and tested on four colored image of $256 \times 256$ size as tabulated in table 1, 2 and 3. The maximum PSNR dB values are satisfied with Two-Circle method. Also, it satisfied minimum frequency change to the steady and stable state of PSNR.

\section{REFERENCES}

[1]. K. H. Talukder and K. Harada, "Haar Wavelet Based Approach for Image Compression and Quality Assessment of Compressed Image", IAENG International Journal of Applied Mathematics, 36-1, IJAM_36_1_9, (2007).

[2]. M. Saraswat, A. K. Wadhwani and M. Dubey, "Compression of Breast Cancer Images by Principal Component Analysis", International journal of Advanced Biological and Biomedical Research, 1, 767-776, (2013).

[3]. J. S. Walker. Wavelet-based Image Compression (2 ${ }^{\text {nd }}$ Edition), University of Wisconsin, Eau Claire (1999).

[4]. S.S.Palewar and Ranjana Shende, "Watermarking Robustness Evaluation Using Enhanced Performance Metrics.", International Journal of Engineering Research \& Technology (IJERT), 2, 2278-0181, (2013).

[5]. N. D.Venkata, T. D. Kite, B. L. Evans, and A. C. Bovik, "Image Quality Assessment Based on a Degradation Model", IEEE Trans. Image Proc. 9, 636-650, (2000). 
[6]. Sungkwang Mun and J. E. Fowler, "BLOCK COMPRESSED SENSING OF IMAGES USING DIRECTIONAL TRANSFORMS", International Conference on Image Processing, Cairo, Egypt, 3021-3024, (2009).

[7]. Yusra A. Y. Al-Najjar and Der Chen Soong, "Comparison of Image Quality Assessment: PSNR, HVS, SSIM, UIQI", International Journal of Scientific \& Engineering Research, 3, 8, 2229-5518, (2012).

[8]. A. Saffor and Abdul Rahman Ramli, "A COMPARATIVE STUDY OF IMAGE COMPRESSION BETWEEN JPEG AND WAVELET", Malaysian Journal of Computer Science, 14, 1, 39-45, (2001).

[9]. N. Salamati and Z. Sadeghipoor, "Compression of Multispectral Images: Color (RGB) plus Near-Infrared (NIR)", (2011).

[10]. N. Dey, A. B. Roy, and S. Dey, "A Novel Approach of Color Image Hiding using RGB Color planes and DWT", International Journal of Computer Applications, 36, 5, 0975 - 8887, (2011). 